\title{
Brain structure and function in people recovering from COVID-19 after hospital discharge or self-isolation: a longitudinal observational study protocol
}

\author{
Bradley J. MacIntosh PhD, Xiang Ji MSc, J. Jean Chen PhD, Asaf Gilboa PhD, Eugenie Roudaia PhD, \\ Allison B. Sekuler PhD, Fuqiang Gao MD, Jordan A. Chad MSc, Aravinthan Jegatheesan PhD, \\ Mario Masellis PhD MD, Maged Goubran PhD, Jennifer Rabin PhD, Benjamin Lam MD, Ivy Cheng MD, \\ Robert Fowler MD, Chris Heyn PhD MD, Sandra E. Black MD, Simon J. Graham PhD
}

\section{Abstract}

Background: The detailed extent of neuroinvasion or deleterious brain changes resulting from COVID-19 and their time courses remain to be determined in relation to "long-haul" COVID-19 symptoms. Our objective is to determine whether there are alterations in functional brain imaging measures among people with COVID-19 after hospital discharge or self-isolation.

Methods: This paper describes a protocol for NeuroCOVID-19, a longitudinal observational study of adults aged 20-75 years at Sunnybrook Health Sciences Centre in Toronto, Ontario, that began in April 2020. We aim to recruit 240 adults, 60 per group: people who contracted COVID-19 and were admitted to hospital (group 1), people who contracted COVID-19 and self-isolated (group 2), people who experienced influenza-like symptoms at acute presentation but tested negative for COVID-19 and self-isolated (group 3, control) and healthy people (group 4, control). Participants are excluded based on premorbid neurologic or severe psychiatric illness, unstable cardiovascular disease, and magnetic resonance imaging (MRI) contraindications. Initial and 3-month follow-up assessments include multiparametric brain MRI and electroencephalography. Sensation and cognition are assessed alongside neuropsychiatric assessments and symptom self-reports. We will test the data from the initial and follow-up assessments for group differences based on 3 outcome measures: MRI cerebral blood flow, MRI resting state fractional amplitude of low-frequency fluctuation and electroencephalography spectral power.

Interpretation: If neurophysiologic alterations are detected in the COVID-19 groups in our NeuroCOVID-19 study, this information could inform future research regarding interventions for long-haul COVID-19. The study results will be disseminated to scientists, clinicians and COVID-19 survivors, as well as the public and private sectors to provide context on how brain measures relate to lingering symptoms.

ARS-CoV-2 and its related illness, COVID-19, can affect the lungs and several other organs, even in people who do not experience the "cytokine storm." Specifically, the brain is susceptible to injury after COVID-19, and studies suggest an increased risk of large-vessel stroke and multiple vascular-territory-related infarcts, for example. ${ }^{1-3}$ Of substantial concern, people may struggle with residual "longhaul" COVID-19 symptoms for weeks or months. Carfi and colleagues $^{4}$ reported that more than $80 \%$ of 143 patients admitted to hospital with acute COVID-19 experienced at least 1 persistent symptom at 36 days after discharge. A larger study showed that $76 \%$ of 1655 patients admitted to hospital reported at least 1 symptom 186 days after discharge. ${ }^{5}$

Long-haul COVID-19 symptoms strongly suggest an impact on the brain, either directly or indirectly. Electronic health records from 62 health care organizations and more than 200000 COVID-19 survivors identified that 1 in 3 people were diagnosed with neurologic or psychiatric conditions in the months after infection. ${ }^{6}$ In a systematic review involving almost 48000 COVID-19 survivors, commonly reported symptoms included fatigue (58\%), headache (44\%), attention disorder (27\%) and dyspnea (24\%). ${ }^{7}$

Those with long-haul symptoms may experience serious symptoms for more than 6 months. In an international survey estimating the prevalence of symptoms over 7 months after the onset of illness, $45 \%$ of the 3762 respondents reported working at a reduced level compared to before their illness, and $22 \%$ were no longer working because of health issues. ${ }^{8}$

Competing interests: See the end of the article.

This article has been peer reviewed.

Correspondence to: Bradley Maclntosh, brad.macintosh@utoronto.ca CMAJ Open 2021 November 30. DOI:10.9778/cmajo.20210023 
The time course of recovery from brain-related long-haul COVID-19 symptoms remains poorly understood. We developed an experimental protocol (NeuroCOVID-19) using multimodality assessments to assess and characterize COVID19 brain changes after a hospital stay or self-isolation for COVID-19. The protocol aims to test the primary hypothesis that groups with COVID-19 show altered brain function, as reflected by vascular and physiologic functional brain measures in relation to control groups. A second hypothesis posits group differences in these functional brain measures between the initial and 3-month follow-up assessments.

\section{Methods}

\section{Study design and setting}

This is a protocol for a longitudinal observational study (NeuroCOVID-19) that aims to recruit 240 adults in equal numbers of males and females. The study began in April 2020.

We will conduct a suite of neurologic tests in participants at the initial assessment and at 3-month follow-up at Sunnybrook Health Sciences Centre, Toronto, Ontario, or remotely via an online meeting platform (depending on the test). Sunnybrook Health Sciences Centre is a large academic hospital housing a research institute with extensive research-dedicated equipment and resources, and is fully affiliated with the University of Toronto. Baycrest Health Sciences is a secondary site for data collection.

The participants are categorized into 4 groups of 60 participants: people who contracted COVID-19 and were admitted to Sunnybrook Health Sciences Centre (group 1), people who contracted COVID-19 and self-isolated (group 2), people who experienced influenza-like symptoms at acute presentation but tested negative for COVID-19 and self-isolated (group 3, control) and healthy people (group 4, control). There is no upper bound on the time lapse between infection and the baseline research visit for the COVID-19 groups because of the need to capture a spectrum of long-haul symptoms and to collect an inclusive sample. ${ }^{9}$ Group 3 participants are assessed within 150 days of their infection. Group 4 participants are recruited to match the other 3 groups on sex, gender and 3 expected age categories (20-39 yr, 40-59 yr and 60-75 yr).

At the initial and 3-month follow-up assessments, brain imaging is performed, and sensory tests, a cognitive test battery and a memory test are administered on site (Table 1). Research staff and participants abide by the hospital infection prevention and control requirements. An emotion test battery, assessment of neuropsychiatric symptoms and self-report questionnaire assessment are administered remotely at baseline and 3-month follow-up.

\section{Participants}

Participants are eligible for inclusion if they are aged 20-75 years and living independently. People are excluded if they have a diagnosis of previous dementia, neurologic disorder, severe psychiatric illness, traumatic brain injury or ongoing unstable cardiovascular disease, or contraindication to magnetic resonance imaging (MRI) (e.g., ferromagnetic implant). Contrast-enhanced MRI is not performed in people with poor kidney function (estimated glomerular filtration rate $<60 \mathrm{~mL} / \mathrm{min}$ per $1.73 \mathrm{~m}^{2}$ ) or at follow-up.

Potentially eligible participants are identified though the emergency department electronic database at Sunnybrook Health Sciences Centre, which is queried weekly by a research staff member. Physicians on the research team are able to facilitate recruitment by helping to identify potentially eligible people. We use monthly meetings, internal emails and telephone calls to communicate between physician referrals and research staff. Community advertisement is being performed

Table 1: Summary of visit assessments for the NeuroCOVID-19 study

\begin{tabular}{|llll|}
\hline Visit no. & \multicolumn{1}{c}{ Location } & \multicolumn{1}{c|}{ Protocol/assessment } & \multicolumn{1}{c|}{ Additional information } \\
\hline 1 & Remote (email or telephone) & $\begin{array}{l}\text { Eligibility, consent, COVID-19 test } \\
\text { documentation }\end{array}$ & $\begin{array}{l}\text { Review inclusion and exclusion criteria, collect } \\
\text { demographic characteristics, medical symptoms and } \\
\text { history, and screen for eligibility based on inclusion/ } \\
\text { exclusion criteria and illness status (24 h before visit 2) }\end{array}$ \\
\hline 2 & On site (in hospital) & $\begin{array}{l}\text { Brain MRI, olfaction, vision, EEG, } \\
\text { NIH Cognition Battery, Mnemonic }\end{array}$ & $\begin{array}{l}\text { Order of brain MRI and other assessments will be } \\
\text { interchanged (pseudorandomized) }\end{array}$ \\
& & Similarity Task & \\
\hline 3 & Remote (teleconference via & NIH Emotion Battery, NIH PROMIS, & \\
& online meeting platform) & $\begin{array}{l}\text { MBI-C, Post-COVID-19 Functional } \\
\text { Status scale, SF-36 }\end{array}$ & \\
\hline 4 (3-mo & On site (in hospital) & Brain MRI, olfaction, vision, EEG, & Contrast agent not injected for MRI \\
follow-up) & & NIH Cognition Battery, mnemonic & \\
& similarity task & \\
\hline 5 (3-mo & Remote (teleconference via & NIH Emotion Battery, NIH PROMIS, & \\
follow-up) & online meeting platform) & MBI-C, Post-COVID-19 Functional & \\
& & Status scale, SF-36 & \\
\hline
\end{tabular}

Note: $\mathrm{EEG}=$ electroencephalography, MBI-C = Mild Behavioral Impairment Checklist, MRI = magnetic resonance imaging, NIH = National Institute of Health, NIH Toolbox = National Institutes of Health Toolbox for the Assessment of Neurological and Behavioral Function, PROMIS = Patient-Reported Outcomes Measurement Information System, SF-36 = 36-Item Short Form Survey. 
through electronic posters. Potentially eligible participants are contacted by telephone or email and screened for eligibility. We obtain informed consent using ethics protocol documents delivered by email.

\section{Data sources}

\section{COVID-19 status}

A COVID-19 diagnosis is determined by a provincially approved facility through a nasopharyngeal or oropharyngeal swab and a subsequent real-time reverse transcription polymerase chain reaction test, according to Public Health Ontario procedures. ${ }^{10}$ Participants provide physical or electronic evidence for their test result from Public Health Ontario or mychart.ca, a service provider of hospital electronic medical information. The test information is shared as photographic evidence with a research staff member. Participants are not infectious during on-site assessments as they arrive at least 14 days after infection, report no contact with anyone with COVID-19 symptoms, and have not travelled internationally within the 14 days before the study visit.

\section{$3 \mathrm{~T}$ magnetic resonance imaging}

Imaging of the brain is performed with the Magnetom Prisma 3 T MRI system (Siemens Healthineers). The MRI protocol includes high-resolution anatomic imaging ( $\mathrm{T}_{1}$-weighted, $\mathrm{T}_{2}$-weighted, fluid-attenuated inversion recovery and susceptibility-weighted imaging) to identify brain lesions and permit analysis of tissue signal intensity and volume.

The following MRI sequences probe brain function. Pseudocontinuous arterial spin labelling MRI is performed with a single postlabelling delay to quantify cerebral blood flow, and resting state functional MRI is performed to evaluate the fractional amplitude of low-frequency fluctuations (fALFFs) in blood-oxygenation-level-dependent signals representative of brain activity (Table 2). Additional functional

Table 2: Sequences, key acquisition parameters, sequence timing and protocol timing of magnetic resonance imaging of the brain*

\begin{tabular}{|c|c|c|}
\hline MRI sequence† & Parameters & $\begin{array}{l}\text { Scan time, } \\
\text { min:s }\end{array}$ \\
\hline $\begin{array}{l}\text { 3-dimensional magnetization-prepared rapid } \\
\text { gradient-echo (MPRAGE) }\end{array}$ & $\begin{array}{l}\text { - } \mathrm{TR} / \mathrm{TE} / \mathrm{TI}=2500 / 4.7 / 1100 \mathrm{~ms} ; \theta=7^{\circ} ; \mathrm{FOV}=256 \times 256 \times 192 \\
\mathrm{~mm} ; 1-\mathrm{mm} \text { isotropic voxels }\end{array}$ & $3: 45$ \\
\hline $\begin{array}{l}\text { 3-dimensional } \mathrm{T}_{2} \text {-weighted fluid-attenuated } \\
\text { inversion recovery (FLAIR) }\end{array}$ & $\begin{array}{l}\text { - } \mathrm{TR} / \mathrm{TE} / \mathrm{TI}=5000 / 388 / 1800 \mathrm{~ms} ; \mathrm{FOV}=256 \times 256 \times 192 \mathrm{~mm} \text {; } \\
\text { 1-mm isotropic voxels }\end{array}$ & $5: 57$ \\
\hline $\begin{array}{l}\text { 3-dimensional pseudocontinuous arterial spin } \\
\text { labelling (pCASL) }\end{array}$ & $\begin{array}{l}\text { - Label duration = } 1500 \mathrm{~ms} \text {; postlabel delay = } 1800 \mathrm{~ms} \\
\text { - } 3 \text {-dimensional echo-planar turbo gradient spin echo readout } \\
\text { - } \mathrm{TR} / \mathrm{TE}_{\text {eff }}=4100 / 37 \mathrm{~ms} ; \mathrm{FOV}=240 \times 240 \times 120 \mathrm{~mm} \\
\text { - 2.5-mm isotropic voxels; } 10 \% \text { oversampling } \\
\text { - Turbo factor = } 14 \text {; background suppression }\end{array}$ & $4: 27$ \\
\hline $\begin{array}{l}\text { 3-dimensional } \mathrm{T}_{2} \text {-weighted sampling perfection } \\
\text { with application-optimized contrasts using } \\
\text { different } \theta \text { evolution (SPACE) }\end{array}$ & $\begin{array}{l}\text { - } \mathrm{TR} / \mathrm{TE}_{\mathrm{eff}}=3200 / 408 \mathrm{~ms} ; \mathrm{FOV}=240 \times 240 \times 176 \mathrm{~mm} ; 0.9-\mathrm{mm} \\
\text { isotropic voxels }\end{array}$ & $3: 42$ \\
\hline $\begin{array}{l}\text { 2-dimensional multislice susceptibility-weighted } \\
\text { imaging (SWI) }\end{array}$ & $\begin{array}{l}\text { - TR/TE }=28 / 20 \mathrm{~ms} ; \mathrm{FOV}=240 \times 240 \times 156 \mathrm{~mm} \\
\text { - Acceleration factor }=2 ; 23.1 \% \text { oversampling } \\
\text { - } 0.625-\mathrm{mm} \times 0.625-\mathrm{mm} \times 3.0-\mathrm{mm} \text { voxels }\end{array}$ & 4:02 \\
\hline $\begin{array}{l}\text { 2-dimensional multislice blood-oxygenation-level- } \\
\text { dependent (BOLD) resting state functional MRI }\end{array}$ & $\begin{array}{l}\text { - 2-dimensional echo-planar imaging; TR/TE }=2130 / 30 \mathrm{~ms} ; \theta=70^{\circ} \\
\text { - FOV }=224 \times 224 \times 140 \mathrm{~mm} ; 3.5-\mathrm{mm} \text { isotropic voxels } \\
\text { - } 250 \text { time points }\end{array}$ & 9:00 \\
\hline $\begin{array}{l}\text { 2-dimensional multislice 3-shell diffusion- } \\
\text { weighted imaging (DWI) }\end{array}$ & $\begin{array}{l}\text { - TR/TE }=4300 / 62 \mathrm{~ms} ; \mathrm{FOV}=240 \times 240 \mathrm{~mm} ; 60 \text { slices; } 2.5-\mathrm{mm} \\
\text { isotropic voxels } \\
\text { - } \mathrm{b}=700,1400,2100 \mathrm{~s} / \mathrm{mm}^{2} \\
\text { - } 30 \text { gradient directions; } 4 \mathrm{~b}=0 \text { values; } 1 \text { average per shell }\end{array}$ & $8: 34$ \\
\hline Contrast agent administration & - & \\
\hline Dynamic susceptibility contrast (DSC) MRI & $\begin{array}{l}\text { - 2-dimensional echo-planar imaging; TR/TE }=1250 / 30 \mathrm{~ms} ; \theta=7^{\circ} \text {; } \\
\text { FOV }=220 \times 220 \times 80 \mathrm{~mm} \\
\text { - 1.74-mm } \times 1.74-\mathrm{mm} \times 4.0 \text {-mm voxels; } 140 \text { time points }\end{array}$ & 3:07 \\
\hline Scanning paused & $\begin{array}{l}\text { - Delay until } 10 \text { min after contrast administration to permit } \\
\text { gadolinium-enhanced } \mathrm{T}_{1} \text {-weighted acquisition }\end{array}$ & $6: 53$ \\
\hline 3-dimensional MPRAGE 10 min after injection & - As above & $3: 45$ \\
\hline Overall & - & $53: 12$ \\
\hline
\end{tabular}


imaging is included in support of the primary aims: 3 -shell diffusion-weighted imaging probes neuroinflammation and tissue microstructural integrity, ${ }^{12}$ and dynamic susceptibility contrast MRI is performed with a common contrast agent (Gadovist [gadobutrol], Bayer) $(1 \mathrm{~mL} / \mathrm{kg}$ intravenously with $25 \mathrm{~mL}$ saline flush at a rate of $5 \mathrm{~mL} / \mathrm{s}$ ) to generate cerebral blood volume maps. Last, 10 minutes after contrast injection, $T_{1}$-weighted MRI is repeated to visualize extravasated contrast agent and potential leakiness of the blood-brain barrier.

\section{Electroencephalography}

A 4-channel wireless electroencephalography (EEG) headband (Muse, InteraXon) is used to record the EEG at rest with eyes closed $(5 \mathrm{~min})$ and eyes open $(5 \mathrm{~min})$. Details of analysis of the EEG data are provided in Appendix 1 (available at www.cmajopen.ca/content/9/4/E1114/suppl/DC1). One auditory oddball EEG task is collected as an additional exploratory test in which participants respond to infrequent target tones and ignore frequent standard tones, and during a visual perceptual task that requires discriminating a contour in a cluttered background of increasing density. ${ }^{13}$

\section{Olfaction}

We use the 40-odorant University of Pennsylvania Smell Identification Test (UPSIT, Sensonics International) to characterize olfactory function relative to normative data. The test yields results ranging from normosmia to severe or total anosmia. ${ }^{14}$

\section{Vision}

As COVID-19 may affect the eye ${ }^{15}$ or neural visual perceptual functions, the Freiburg Vision Test ${ }^{16}$ is administered to measure far visual acuity and vernier acuity, and binocular contrast sensitivity is measured with the Pelli-Robson contrast sensitivity test. ${ }^{17}$

\section{Cognition}

The NIH (National Institutes of Health) Toolbox for the Assessment of Neurological and Behavioral Function full Cognition Battery are administered on site via an iPad app..$^{18}$ The battery comprises 7 instruments assessing processing speed, working memory, executive function and episodic memory, which together provide composite scores of crystalized cognition and fluid cognition, and a total cognition score. We use the Mnemonic Similarity Task to assess memory, as this test is sensitive to age-related memory decline and functional brain connectivity. ${ }^{19}$

\section{Other assessments}

The full NIH Emotion Battery, ${ }^{20}$ a modified Mild Behavioral Impairment Checklist ${ }^{21}$ and the NIH Patient-Reported Outcomes Measurement Information System (PROMIS) are administered during a one-on-one online meeting on a secure Zoom platform. The PROMIS includes short-form scales to measure fatigue, dyspnea, cognitive function and sleep disturbance symptoms. General functional status is assessed with the Post-COVID-19 Functional Status scale, ${ }^{22}$ and the 36-Item Short Form Survey assesses health-related quality of life $^{23}$ (Appendix 1). These data are entered directly into a REDCap database by a research staff member (X.J., E.R. or S.J.G.) and verified for accuracy by another staff member (X.J., E.R. or S.J.G.).

\section{Statistical analysis}

Using a scoring sheet adapted from a previous visual rating rubric, ${ }^{24} 2$ neuroradiologists score anatomic brain MRI scans to visualize lesions of presumed vascular origin, cerebral microbleeds and inflammation in brain tissue..$^{25} \mathrm{We}$ expect that a proportion of people recovering from COVID-19 as well as control participants will show vascular lesions related to age and vascular risk factors on MRI. Furthermore, pathogens (e.g., bacterial, viral) can invade the central nervous system, and this may be reflected in the control participants who experienced influenza-like symptoms. ${ }^{26}$ Diffusion-weighted images are conducive to conventional maps of mean diffusivity or restricted diffusion. Quantitative anatomic volumetric estimation is conducted with semiautomatic Sunnybrook software as a supplementary analysis. ${ }^{27}$

We will use freeware functional neuroimaging packages to obtain cerebral blood flow ${ }^{28}$ and fALFF ${ }^{29}$ outcome maps; participant data will be coregistered to a standard brain atlas coordinate space. The study is powered to detect omnibus group differences on the basis of the arterial spin labelling (cerebral blood flow) and functional MRI (fALFF) functional brain maps that will be observed in predefined regions of interest. We will also perform equivalent statistical testing using voxel-wise analysis, because potential findings will need to be examined thoroughly throughout the entire brain. Additional details on these measures are provided in Appendix 1.

We will use a one-way analysis of variance model for hypothesis testing. We performed a preliminary power analysis assuming $\alpha=0.01$, power $=0.80$ and an effect size of 0.26 ( $G^{*}$ Power software); a total of 240 participants are required per group (30 men and 30 women in each of the 4 groups) to reach a critical $F$ value of 3.9, with 3 and 236 degrees of freedom. We will use age and sex as the first set of covariates. Post hoc analyses will evaluate pair-wise group comparisons (e.g., group 2 v. group 3; group 2 v. group 4), and age- and sex-stratified subgroups.

Electroencephalography at rest will be characterized with power spectral density across frequency bands, ${ }^{30}$ and we will use these measures for hypothesis testing. Additional analysis of the EEG data will include examination of signal complexity, ${ }^{31}$ as it is known to be sensitive to brain injury. ${ }^{32}$ Eventrelated potentials in response to auditory oddball and visual stimuli will be used to probe possible brain stem dysfunction associated with cognitive impairment and possible delays in visual perceptual processes.

Long-haul COVID-19 symptoms are characterized by olfaction, domain-specific cognitive impairment and neuropsychiatric measures. ${ }^{433}$ We use existing scoring methods to obtain standardized scores for olfaction, NIH Toolbox, PROMIS and 36-Item Short Form Survey measures relative to normative data. We will use a linear mixed-effects model to 
test whether longitudinal changes in these measures explain variance in cerebral blood flow, fALFF and EEG functional brain outcomes from the initial to the 3-month assessment. We will use a multiple imputation method to account for data lost to follow-up and to mitigate bias. ${ }^{34}$

\section{Ethics approval}

This study protocol was approved by the Sunnybrook Health Sciences Centre Research Ethics Board and the Baycrest Health Sciences Research Ethics Board.

\section{Interpretation}

In this longitudinal observational study assessing and characterizing COVID-19 brain changes after a hospital stay or selfisolation for COVID-19, the inclusion of participants who experienced influenza-like symptoms at acute presentation but tested negative for COVID-19 and self-isolated (group 3, control) will enable us to account for other typical viral pathogens, and the inclusion of healthy participants (group 4, control) will enable us to account for the societal effects of the pandemic. $26,35,36$

At the time of writing, 73 participants ( 8 in group 1, 50 in group 2 and 15 in group 3 ) had had an initial assessment, and 61 (6, 42 and 13, respectively) had completed the 3-month follow-up assessment; 3 participants declined follow-up because of MRI-related anxiety.

Ethical aspects and knowledge translation need to be considered in implementing this protocol. One ethics consideration involves incidental findings, which are likely to occur. To address brain imaging findings such as excessive cerebral microbleeds, for example, procedures have been implemented to initiate follow-up with diagnostic radiologic imaging. Clinician-researchers from the NeuroCOVID-19 team are prepared to provide clinical advice and care, as required, for participants for whom additional clinical services such as cognitive neurology or psychiatry may be warranted.

The overall implication is that the study will provide detailed characterization of the functional neuroimaging correlates of long-haul COVID-19 symptoms and subtypes, which will assist in the development and implementation of effective treatments for these conditions. To raise awareness of these research outputs among scientists, clinicians, and the public and private sectors, NeuroCOVID-19 team members will participate in academic conferences and national forums related to COVID-19, as well as news and social media outreach. Research newsletters will also convey timely information to study participants.

Knowledge exchange is possible through scientific collaboration or adoption of the protocol at other sites. For example, high-spatial-resolution $3 \mathrm{~T}$ MRI permits more detailed characterization of brain anatomy than is achieved with typical clinical imaging. Furthermore, functional brain maps collected under a resting state will be deployed to increase sensitivity to altered brain physiology. Aspects of the sensory, behavioural and EEG tests are conducive to mobile or remote assessment.

\section{Limitations}

Diagnostic certainty of COVID-19 status is limited by the sensitivity and specificity of the polymerase chain reaction tests. Blood biomarkers of immunity status and other factors would provide important complementary information and can be added to the protocol in the future.

Recruitment bias cannot be ruled out. In addition, recruitment into the COVID-19 groups does not include an upper bound on the number of days since infection. Although this provides a more inclusive sample, the effect sizes may be reduced in the group comparisons as a consequence. The age of inclusion is wide, which introduces within-group agerelated variability, but this also provides an opportunity to better understand the influence of young, mid-life and older age demographic characteristics on COVID-19 sequelae. A UK surveillance study showed that young and middle-aged COVID-19 survivors were more likely to report neuropsychiatric issues than cerebrovascular issues. ${ }^{37}$ Hence, the functional imaging measures in NeuroCOVID-19 may help to elucidate this phenomenon by age-stratified post hoc analyses.

Prepandemic brain measures, which other studies have been able to achieve, ${ }^{38}$ are not part of the current protocol.

The 3-month follow-up visit after the initial visit was a pragmatic choice to capture a recovery point that coincides roughly with 6-12 months from the time of infection for the study participants with lingering COVID-19 symptoms. The protocol can easily be extended to facilitate additional longitudinal assessments.

\section{Conclusion}

The detailed extent of neuroinvasion or deleterious brain changes resulting from COVID-19 and their time courses remain to be determined in relation to long-haul COVID-19 symptoms. The NeuroCOVID-19 protocol is designed to address this issue through innovative methodology. We expect that it will be possible to translate the knowledge gained from NeuroCOVID-19 into effective, targeted interventions for people with specific long-haul COVID-19 symptoms.

\section{References}

1. Nannoni S, de Groot R, Bell S, et al. Stroke in COVID-19: a systematic review and meta-analysis. Int 7 Stroke 2021;16:137-49.

2. Merkler AE, Parikh NS, Mir S, et al. Risk of ischemic stroke in patients with coronavirus disease 2019 (COVID-19) vs patients with influenza. $\mathcal{F} A M A$ Neurol 2020;77:1-7.

3. Katz JM, Libman RB, Wang JJ, et al. Cerebrovascular complications of COVID-19. Stroke 2020;51:e227-31.

4. Carfi A, Bernabei R, Landi F; Gemelli Against COVID-19 Post-Acute Care Study Group. Persistent symptoms in patients after acute COVID-19. FAMA 2020;324:603-5.

5. Huang C, Huang L, Wang Y, et al. 6-month consequences of COVID-19 in patients discharged from hospital: a cohort study. Lancet 2021;397:220-32.

6. Taquet M, Geddes JR, Husain M, et al. 6-month neurological and psychiatric outcomes in 236379 survivors of COVID-19: a retrospective cohort study using electronic health records. Lancet Psychiatry 2021;8:416-27.

7. Lopez-Leon S, Wegman-Ostrosky T, Perelman C, et al. More than 50 longterm effects of COVID-19: a systematic review and meta-analysis. MedRxiv [preprint] 2021 Jan 30;2021.01.27.21250617. doi: 10.1101/2021.01.27.21250617.

8. Davis HE, Assaf GS, McCorkell L, et al. Characterizing long COVID in an international cohort: 7 months of symptoms and their impact. MedRxiv [preprint] 2021 Apr. 5. doi: https://doi.org/10.1101/2020.12.24.20248802. Available: http:// medrxiv.org/lookup/doi/10.1101/2020.12.24.20248802 (accessed 2021 May 12).

9. Witham MD, Anderson E, Carroll CB, et al. Ensuring that COVID-19 research is inclusive: guidance from the NIHR INCLUDE project. BMF Open 2020;10:e043634. 
10. Coronavirus disease 2019 (COVID-19) - PCR. Toronto: Public Health Ontario. Available: https://www.publichealthontario.ca/en/laboratory-services/ test-information-index/covid-19 (accessed 2021 Jan. 19).

11. Alsop DC, Detre JA, Golay X, et al. Recommended implementation of arterial spin-labeled perfusion MRI for clinical applications: a consensus of the ISMRM Perfusion Study Group and the European consortium for ASL in Dementia. Magn Reson Med 2015;73:102-16.

12. Steardo L, Steardo L, Zorec R, et al. Neuroinfection may contribute to pathophysiology and clinical manifestations of COVID-19. Acta Physiol (Oxf) 2020;229:e13473.

13. Roudaia E, Bennett PJ, Sekuler AB. Contour integration and aging: the effects of element spacing, orientation alignment and stimulus duration. Front Psychol 2013;4:356.

14. Moein ST, Hashemian SM, Mansourafshar B, et al. Smell dysfunction: a biomarker for COVID-19. Int Forum Allergy Rhinol 2020;10:944-50.

15. Lee YH, Kim YC, Shin JP. Characteristics of ocular manifestations of patients with coronavirus disease 2019 in Daegu Province, Korea. 7 Korean Med Sci 2020;35:e322.

16. Bach $M$. The Freiburg Visual Acuity test - automatic measurement of visual acuity. Optom Vis Sci 1996;73:49-53.

17. Elliott DB, Sanderson K, Conkey A. The reliability of the Pelli-Robson contrast sensitivity chart. Opbthalmic Physiol 1990;10:21-4.

18. Weintraub S, Dikmen SS, Heaton RK, et al. Cognition assessment using the NIH Toolbox. Neurology 2013;80(Suppl 3):S54-64.

19. Stark SM, Kirwan CB, Stark CEL. Mnemonic Similarity Task: a tool for assessing hippocampal integrity. Trends Cogn Sci 2019;23:938-51.

20. Salsman JM, Butt Z, Pilkonis PA, et al. Emotion assessment using the NIH Toolbox. Neurology 2013;80(Suppl 3):S76-86.

21. Ismail Z, Agüera-Ortiz L, Brodaty H, et al. The Mild Behavioral Impairment Checklist (MBI-C): a rating scale for neuropsychiatric symptoms in predementia populations. 7 Alzheimers Dis 2017;56:929-38.

22. Klok FA, Boon GJAM, Barco S, et al. The Post-COVID-19 Functional Status scale: a tool to measure functional status over time after COVID-19. Eur Respir 7 2020;56:2001494.

23. Ware JE, Sherbourne CD. The MOS 36-item short-form health survey (SF36). I. Conceptual framework and item selection. Med Care 1992;30:473-83.

24. Staals J, Makin SDJ, Doubal FN, et al. Stroke subtype, vascular risk factors, and total MRI brain small-vessel disease burden. Neurology 2014;83:1228-34.

25. Netland J, Meyerholz DK, Moore S, et al. Severe acute respiratory syndrome coronavirus infection causes neuronal death in the absence of encephalitis in mice transgenic for human ACE2. 7 Virol 2008;82:7264-75.

26. Dando SJ, Mackay-Sim A, Norton R, et al. Pathogens penetrating the central nervous system: infection pathways and the cellular and molecular mechanisms of invasion. Clin Microbiol Rev 2014;27:691-726.

27. Ramirez J, Gibson E, Quddus A, et al. Lesion Explorer: a comprehensive segmentation and parcellation package to obtain regional volumetrics for subcortical hyperintensities and intracranial tissue. Neuroimage 2011;54:963-73.

28. Mutsaerts HJMM, Petr J, Groot P, et al. ExploreASL: an image processing pipeline for multi-center ASL perfusion MRI studies. Neuroimage 2020;219:117031.

29. Zou QH, Zhu CZ, Yang Y, et al. An improved approach to detection of amplitude of low-frequency fluctuation (ALFF) for resting-state fMRI: fractional ALFF. 7 Neurosci Methods 2008;172:137-41.

30. Hashemi A, Pino LJ, Moffat G, et al. Characterizing population EEG dynamics throughout adulthood. eNeuro 2016;3:ENEURO.0275-16.2016.

31. Costa M, Goldberger AL, Peng CK. Multiscale entropy analysis of complex physiologic time series. Phys Rev Lett 2002;89:068102.

32. Raja Beharelle A, Kovacevic N, McIntosh AR, et al. Brain signal variability relates to stability of behavior after recovery from diffuse brain injury. Neuroimage 2012;60:1528-37.

33. Michelen M, Manoharan L, Elkheir N, et al. Characterising long COVID: a living systematic review. BM7 Glob Health 2021;6:e005427.

34. Churchill NW, Hutchison MG, Graham SJ, et al. Neurometabolites and sport-related concussion: from acute injury to one year after medical clearance. Neuroimage Clin 2020;27:102258.

35. Dubey S, Biswas P, Ghosh R, et al. Psychosocial impact of COVID-19. Diabetes Metab Syndr 2020;14:779-88.

36. Amin-Chowdhury Z, Harris RJ, Aiano F, et al. Characterising post-COVID syndrome more than 6 months after acute infection in adults; prospective longitudinal cohort study, England. MedRxiv [preprint] 2021 Apr. 16. Available: http:// medrxiv.org/lookup/doi/10.1101/2021.03.18.21253633 (accessed 2021 July 28).

37. Varatharaj A, Thomas N, Ellul MA, et al. Neurological and neuropsychiatric complications of COVID-19 in 153 patients: a UK-wide surveillance study. Lancet Psychiatry 2020;7:875-82.

38. Douaud G, Lee S, Alfaro-Almagro F, et al. Brain imaging before and after COVID-19 in UK Biobank. MedRxiv [preprint] 2021 June 15. doi: https://doi. org/10.1101/2021.06.11.21258690.

Competing interests: Sandra Black reports payments for contract research to her institution from GE Healthcare, Eli Lilly and Company, Biogen, Genentech, Optina Diagnostics and Roche; consulting fees and payments related to an advisory board from Roche; and payments related to an advisory board, a speaker panel, talks and an educational session from Biogen. There were peer-reviewed grants to her institution from the Ontario Brain Institute,
Canadian Institutes of Health Research, Leducq Foundation, Heart and Stroke Foundation of Canada, National Institutes of Health, Alzheimer's Drug Discovery Foundation, Brain Canada, Weston Brain Institute, Canadian Partnership for Stroke Recovery, Canadian Foundation for Innovation, Focused Ultrasound Foundation, Alzheimer's Association US, Department of National Defence, Montreal Medical International - Kuwait, Queen's University, Compute Canada Resources for Research Groups, CANARIE and Networks of Centres of Excellence of Canada. She has participated on a data safety monitoring board or advisory board for the Conference Board of Canada, World Dementia Council and University of Rochester. She has contributed to the mission and scientific leadership of the Small Vessel VCID Biomarker Validation Consortium, National Institute of Neurological Disorders and Stroke. No other competing interests were declared.

Affiliations: Hurvitz Brain Sciences Program (MacIntosh, Gao, Masellis, Goubran, Lam, Heyn, Black, Graham), Physical Sciences Platform (MacIntosh, Jegatheesan, Goubran, Graham), Evaluative Clinical Sciences, Integrated Community Program (Cheng), Harquail Centre for Neuromodulation (Rabin) and Evaluative Clinical Sciences, Trauma, Emergency \& Critical Care Research Program (Fowler), Sunnybrook Research Institute; Department of Medical Biophysics (MacIntosh, Chen, Chad, Jegatheesan, Goubran, Graham), University of Toronto; LC Campbell Cognitive Neurology Research Group (Ji, Gao, Masellis, Lam, Black), Sunnybrook Hospital; Rotman Research Institute (Chen, Gilboa, Roudaia, Sekuler, Chad), Baycrest Health Sciences; Division of Neurology (Masellis, Rabin, Lam, Black), Department of Medicine, University of Toronto; Rehabilitation Sciences Institute (Rabin), Department of Medical Imaging (Heyn) and Department of Psychology (Gilboa, Sekuler), University of Toronto; Department of Medicine (Cheng, Fowler), University of Toronto, Sunnybrook Health Sciences Centre, Toronto, Ont.; Department of Psychology, Neuroscience \& Behaviour (Sekuler), McMaster University, Hamilton, Ont.

Contributors: Bradley MacIntosh, Xiang Ji, Jean Chen, Asaf Gilboa, Eugenie Roudaia and Allison Sekuler are first authors. Bradley MacIntosh, Jean Chen, Asaf Gilboa, Eugenie Roudaia, Allison Sekuler, Benjamin Lam and Simon Graham conceived of the study. Xiang Ji, Ivy Cheng, Robert Fowler, Chris Heyn and Sandra Black contributed to the study design. Xiang Ji is leading data collection. Bradley MacIntosh, Xiang Ji, Jean Chen, Asaf Gilboa, Eugenie Roudaia, Allison Sekuler, Benjamin Lam and Simon Graham drafted the manuscript. Xiang Ji, Fuqiang Gao, Jordan Chad, Aravinthan Jegatheesan, Mario Masellis, Maged Goubran, Jennifer Rabin, Ivy Cheng, Robert Fowler, Chris Heyn and Sandra Black revised the manuscript critically for important intellectual content. All of the authors approved the final version to be published and agreed to be accountable for all aspects of the work.

Funding: This study is funded by the Sunnybrook Foundation, the Dr. Sandra Black Centre for Brain Resilience \& Recovery, Sunnybrook Health Sciences Centre, and a Canadian Institutes of Health Research Operating Grant on Emerging COVID-19 Research Gaps and Priorities (grant number 410015282).

Content licence: This is an Open Access article distributed in accordance with the terms of the Creative Commons Attribution (CC BY-NC-ND 4.0) licence, which permits use, distribution and reproduction in any medium, provided that the original publication is properly cited, the use is noncommercial (i.e., research or educational use), and no modifications or adaptations are made. See: https://creativecommons.org/licenses/by-nc-nd/4.0/.

Data sharing: Data produced from this study will abide by the joint statement on sharing research data and findings relevant to the novel coronavirus outbreak (https://wellcome.org/coronavirus-covid-19/open -data); namely: reports and publications stemming from this research will be open access, and portions of the data (organized as metadata or aggregate forms of raw data) will be available to others through data-sharing agreements with Sunnybrook Research Institute via secure Web portal.

Acknowledgements: The authors gratefully acknowledge the contributions of magnetic resonance imaging technologists Garry Detzler and Ruby Endre. They thank Ellen Cohen, Masud Hussain, Haddas Grosbein and Devin Sodums for their integral role in recruitment and data collection.

Supplemental information: For reviewer comments and the original submission of this manuscript, please see www.cmajopen.ca/content/9/4/ E1114/suppl/DC1. 